First publ. in: Behavioral and Brain Sciences 23 (2000), 3, pp. 406-407

\section{Physiological units and behavioral elements: Dynamic brains relate to dynamic behavior}

\author{
Andreas Keil and Thomas Elbert \\ Department of Psychology, University of Konstanz, Box D25, D-78457, \\ Konstanz, Germany Andreas.Keil@Uni-Konstanz.de \\ Thomas.Elbert@Uni-Konstanz.de \\ www.clinical-psychology.uni-Konstanz.de/
}

Abstract: Nunez is to be applauded for putting forward a theoretical brain model. In order to improve any model it needs to be experimentally testable. The model presented in the target article suffers from insufficient clarity as to how new experimental designs could be derived. This is a consequence of neglecting the purpose of the brain, which is to produce effective and adaptive behavior. It might be possible to overcome this drawback by including Hebb-based modeling.

A major problem in the interpretation of EEG/MEG findings has been the lack of a coherent theoretical framework within which to articulate and test the results from experimental studies. Paul Nunez provides an interesting approach for the interpretation and analysis of electromagnetic measures of neural mass activity. This is the more remarkable as reference to dynamical aspects of the signals is usually sparse in ERP/EEG literature. Furthermore, the relation between specific experimental findings and brain theory has been examined only sporadically. This strongly contrasts with the fact that the processes underlying the scalp-recorded voltage modulations remain unclear unless examined on the ground of an appropriate theoretical foundation. What would the main characteristics of such a theoretical account be? We shall confine our comments to two points that may be complementary to some of the main issues raised in the target article.

First, we would like to address the issue of experimental para- digm selection and its consequences for theory-guided predictions on EEG parameters. In the target article and his 1995 book, Nunez describes a number of studies, the results of which can be explained by local/global theory in a post-hoc manner. In addition, several predictions are made for spectral characteristics of spontaneous EEG in distinct brain states such as sleep stages. The target article mentions the fact that the predictions made on the basis of the local equations may be hampered by global effects and vice versa. Nunez acknowledges that his "theory is silent on most cognitive questions because it was developed specifically to describe EEG, not behavioral or cognitive correlates" (sect. 2.7, para. 2). However, without further specification and more precise relation to variables on a behavior or psychological level, this approach seems to have limited applicability for designing meaningful studies of brain functioning. What is needed is a theoretical account of brain-behavior relations in terms of task-specific assumptions and predictions, being embedded in a behavioral theory. Of course, the selection of appropriate algorithms is not trivial, because a number of alternate mathematical models are also able to predict the cortical dynamics as recorded by EEG (e.g., Elbert \& Rockstroh 1987; Skarda \& Freeman 1987). One example for a theoretical framework that allows both for the construction of experimental paradigms and selection of relevant parameters may be the work by Haken, Kelso, and collaborators, referred to in the target article. Their synergetic approach incorporates predictions for both behavioral and neuronal dynamics during performance of well-defined perceptual and motor tasks (e.g., Kelso et al. 1995). In order to test these predictions, experimental designs have been developed that allow for monitoring phase transitions (i.e., qualitative changes between system states) using behavioral measures such as self-report or electromyogram on the one hand as well as electrocortical recordings on the other. Thus, a dynamic perspective is not only applied to brain parameters, but also to the experimental design.

Second, an extension of the Nunez model might be achieved if our understanding can be integrated regarding how Hebbian cell assemblies are sculptured within a highly interconnected neuropil by the forces of stimulus-evoked activity. These forces include experience-driven synaptic plasticity (synapse formation, spine density, and alterations in dendritic length) and the competition of previously formed cell assemblies. The power of the Hebbian idea derives from the connection between physiological units and behavioral elements. A cell assembly can be spread across large cortical areas (Pulvermüller et al. 1999). The meaning and qualitative nature of an event, an idea, an emotion, or a percept, are reflected in the local topography of its connections and firing patterns, so to speak, in the topographical "Gestalt" of an assembly in its phase space and not in the properties of its parts, cells, or transmitters. A model based on such considerations has been presented by the recent target article of Pulvermüller (1999). The specificity of an assembly is best reflected in the spatial distribution and frequency of fast-changing electrical activities, such as the EEG and event-related components. Meanwhile, a large number of studies have converged to show a strong correlation between highfrequency brain activity and experimental manipulations. The measures used include single-cell recordings, local field potentials, electrocorticogram, EEG spectral power, and EEG coherence (Keil et al. 1999; Pulvermüller et al. 1999; Tallon-Baudry \& Bertrand 1999). Nunez's local/global theory could contribute to these findings, providing an approach to quantify and predict interactions between these scales, for example, as proposed in Equation 5 of the target article.

The need for an integration of approaches such as Nunez's and aspects of Hebbian plasticity and self-organization is evident: Any physiological activity has to be nonlinear and nonrandom, because living biological systems can only function if held within certain activity limits through feedback of the ongoing activity. Consequently, cell assemblies, and therefore the EEG and ERP, may have properties of deterministic chaos (for review see, e.g., Elbert et al. 1994). Skarda and Freeman (1987), for instance, have shown 
in the olfactory system of the rabbit that chaos becomes more prevalent when there is competition among parts of assemblies, or among several assemblies. Why shouldn't similar mechanisms hold for the human neocortex? 